\title{
Numerical evolution of shocks in the interior of Kerr black holes
}

\author{
Paul M. Chesler \\ Black Hole Initiative, Harvard University, Cambridge, Massachusetts 02138, USA \\ Erik Curiel ${ }^{\dagger}$ \\ Munich Center for Mathematical Philosophy, Ludwig-Maximilians-Universität, \\ Ludwigstraß 31, 80539 München, Germany and Black Hole Initiative, Harvard University, \\ Cambridge, Massachusetts 02138, USA \\ Ramesh Narayan \\ Black Hole Initiative, Harvard University, Cambridge, Massachusetts 02138, USA
}

(Received 15 October 2018; published 17 April 2019)

\begin{abstract}
We numerically solve Einstein's equations coupled to a scalar field in the interior of Kerr black holes. We find shock waves form near the inner horizon. The shocks grow exponentially in amplitude and need not be axisymmetric. Observers who pass through the shocks experience exponentially large tidal forces and are accelerated exponentially close to the speed of light.
\end{abstract}

DOI: 10.1103/PhysRevD.99.084033

\section{INTRODUCTION}

The no-hair theorem postulates that the exterior geometry of black holes is completely described by the black hole's mass, charge and angular momentum via the KerrNewman metric. The origin of this lies in the fact that perturbations near the event horizon can either be absorbed by the event horizon or radiated to infinity, allowing the near horizon geometry to relax. In fact the no-hair theorem should also hold just inside the event horizon as well, since just inside the event horizon of the Kerr-Newman metric, all light rays propagate deeper into the interior. However, inside the inner horizon of the Kerr-Newman geometry, light rays need not propagate deeper into the interior, meaning there is no mechanism for perturbations to relax and the no-hair theorem does not apply there.

The interior geometry of black holes has been most widely studied for Reissner-Nordström black holes [1-15]. This is due to the fact that one can impose spherical symmetry, greatly simplifying calculations. One striking result is the presence of gravitational shock waves [13,15], which form along the outgoing branch of the inner horizon [16]. The formation mechanism of the shocks lies in the fact that from the perspective of infalling observers, outgoing radiation in the black hole is blueshifted by a factor of $e^{\kappa v}$, with $v$ being advanced time and $\kappa$ the surface gravity of the inner horizon, and localized to a ball whose surface approaches the inner horizon radius as $v \rightarrow \infty$. This means

\footnotetext{
*pchesler@g.harvard.edu erik@strangebeautiful.com

"rnarayan@cfa.harvard.edu
}

that upon passing through the inner horizon radius-the location of the shock-infalling observers will measure a Riemann tensor of order $e^{2 \kappa v}$ and therefore experience exponentially large gravitational and tidal forces. The gravitational kick imparted by the shock results in infalling observers being exponentially accelerated inwards towards the central singularity. For a solar mass black hole, the proper time interval between passing through the shock and approaching the singularity becomes Planckian milliseconds after the black hole forms. Hence infalling observers encounter a curvature brick wall at the shock, where the curvature increases from its approximate ReissnerNordström value to arbitrarily large values (which depend on their precise trajectory near the singularity) over Planckian proper times.

For Kerr black holes (or more generally Kerr-Newman black holes), infalling observers will also see outgoing radiation in the black hole blueshifted by $e^{\kappa v}$. Hence, it is natural to expect gravitational shocks to form in the interior of rotating black holes [15]. In the present work we study the evolution of shocks in Kerr black holes by numerically solving Einstein's equations coupled to a scalar field. We study both axisymmetric and nonaxisymmetric solutions. Like Reissner-Nordström black holes, we find shocks form near the outgoing leg of the inner horizon. In addition to solving Einstein's equations numerically, we also solve them analytically with a derivative expansion in the vicinity of the shocks and find excellent agreement with the numerics. Like Reissner-Nordström black holes, we find that shocks in Kerr black holes dramatically affect infalling geodesics passing through them. In particular, 
infalling timelike observers passing through the shocks are accelerated exponentially close to the speed of light and experience exponentially large tidal forces.

\section{SETUP}

We numerically solve Einstein's equations coupled to a massless real scalar field $\Psi$. The equations of motion read

$$
R^{\mu \nu}-\frac{1}{2} R g^{\mu \nu}=8 \pi T^{\mu \nu},
$$

and

$$
D^{2} \Psi=0
$$

where $D_{\mu}$ is the covariant derivative operator and

$$
T_{\mu \nu}=D_{\mu} \Psi D_{\nu} \Psi-\frac{1}{2} g_{\mu \nu}(D \Psi)^{2},
$$

is the scalar stress tensor.

Our numerical evolution scheme is detailed in Ref. [17]. Here we outline the salient details. We employ a characteristic evolution scheme where the metric takes the form

$$
\begin{aligned}
d s^{2}= & -2 A d v^{2}+2 d v d \lambda \\
& +r^{2} h_{a b}\left(d x^{a}-F^{a} d v\right)\left(d x^{b}-F^{b} d v\right),
\end{aligned}
$$

with $x^{a}=\{\theta, \varphi\}$ where $\theta$ is the polar angle and $\varphi$ is the azimuthal angle. The two-dimensional angular metric $h_{a b}$ satisfies det $h_{a b}=\sin ^{2} \theta$, meaning the function $r$ is an areal coordinate. Lines of constant time $v$ and angles $\theta, \varphi$ are radial null infalling geodesics. The radial coordinate $\lambda$ is an affine parameter for these geodesics. Correspondingly, the metric (4) is invariant under the residual diffeomorphism

$$
\lambda \rightarrow \lambda+\xi(v, \theta, \varphi),
$$

where $\xi$ is arbitrary. We fix $\xi$ such that the inner horizon of the stationary Kerr geometry is located at

$$
\lambda=\lambda_{-}=1 .
$$

Requisite initial data at $v=0$ consists of the scalar field $\Psi$ and the angular metric $h_{a b}$. The remaining components of the metric are determined by initial value constraint equations [17]. Perhaps the most natural initial data is that where a rotating black hole is formed dynamically via gravitational collapse. Another option would be to start with Kerr initial data and allow infalling radiation to perturb the geometry inside the event horizon at $\lambda=\lambda_{+}$. A third option is to start with Kerr initial data and add a perturbation inside the event horizon. To study the evolution of shocks, it is sufficient to consider the last option, as this offers several computational advantages. First, limiting perturbations to the interior of the black hole means that one can restrict the computational domain to the interior of the black hole. Second, since no energy or angular momentum can be radiated to infinity, the mass and spin of the black hole remain constant. Because the geometry outside the inner horizon should be stable, this means that at late times the position of the inner horizon must approach that of the unperturbed Kerr geometry at $\lambda=1$. In our coordinate system this ultimately means that at late times one must have $A \rightarrow 0$ at $\lambda=1$. Having the inner horizon approach constant $\lambda$ is useful, since shocks are expected to form there.

We employ the Kerr metric for initial $h_{a b}$. For initial scalar data we choose

$\Psi=\frac{1}{50} e^{-\left(\lambda-\lambda_{0}\right)^{2} / 2 \sigma^{2}}\left\{1+\zeta \operatorname{Re}\left[y^{10}(\theta, \varphi)+y^{11}(\theta, \varphi)\right]\right\}$,

where $y^{\ell m}$ are spherical harmonics and $\zeta$ is a parameter controlling the degree of nonaxisymmetry in the initial data. We choose $\lambda_{0}$ and $\sigma$ such that $\Psi$ is localized between $\lambda=1$ and $\lambda=\lambda_{+}$and exponentially small at our outer computational boundary. We fix the Kerr mass parameter $M=1$ and spin $a=0.9,0.95$ and 0.99 and evolve until $v_{\max }=9 / \kappa$ where $\kappa$ is the surface gravity of the inner horizon of the unperturbed Kerr black hole. For $a=0.9$, 0.95 we set $\left(\lambda_{0}, \sigma\right)=(1.05,1 / 150)$ while for $a=0.99$ we set $\left(\lambda_{0}, \sigma\right)=(1.01,1 / 500)$. For axisymmetric initial data we set $\zeta=0$ and for nonaxisymmetric initial data we set $\zeta=1 / 4$.

We employ a time-dependent radial computational domain $\lambda_{\min }(v) \leq \lambda \leq \lambda_{\max }(v) . \lambda_{\min }(v)$ and $\lambda_{\max }(v)$ will be surfaces which at late times asymptote to $\lambda=1$ from below and above respectively. See Fig. 1 for a Penrose diagram illustrating our computational domain. We choose

$$
\frac{d \lambda_{\max }}{d v}=\left.\min _{\theta, \varphi} A\right|_{\lambda=\lambda_{\max }}, \quad \frac{d \lambda_{\min }}{d v}=\left.\max _{\theta, \varphi} A\right|_{\lambda=\lambda_{\min }} .
$$

These choices mean that the surfaces $\lambda_{\min }(v)$ and $\lambda_{\max }(v)$ are either spacelike or null. This in turn means that no information can propagate from inside $\lambda_{\min }$ through $\lambda_{\min }$. At $\lambda_{\max }$, where the scalar field is exponentially small, we impose the boundary condition that the geometry is that of Kerr. This is allowed since no signal from inside $\lambda_{\max }$ can ever reach $\lambda_{\max }$.

Our discretization scheme is nearly identical to that in Ref. [18]. To discretize the equations of motion we make a linear change of coordinates from $\lambda$ to $z \in(-1,1)$ via

$$
\lambda=a(v) z+b(v),
$$

where

$$
a(v)=\frac{1}{2}\left(\lambda_{\max }(v)-\lambda_{\min }(v)\right),
$$




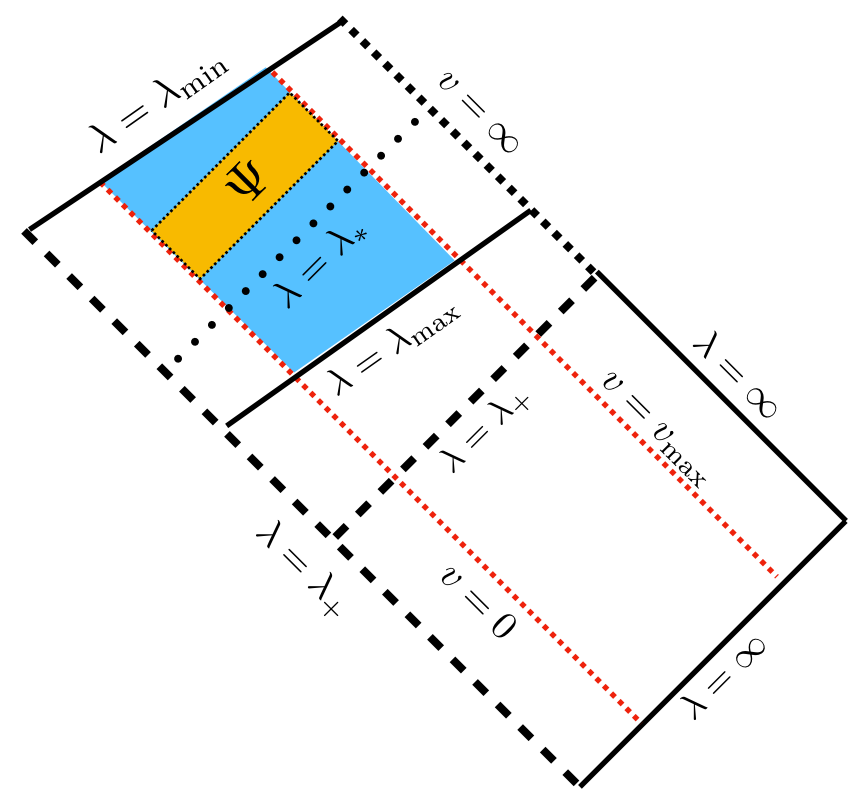

FIG. 1. A Penrose diagram showing our computational domain, which is represented by the blue shaded region. Our radial coordinate $\lambda$ is the affine parameter for infalling radial geodesics, two of which are shown as the red dashed lines. The event horizon is located at $\lambda=\lambda_{+}$. The scalar field $\Psi$ (yellow shaded region) is localized inside the event horizon between $\lambda_{\min }(v)$ and $\lambda_{\max }(v)$, which are spacelike surfaces which at late times asymptote to $\lambda=1$, the location of the inner horizon of the Kerr geometry. The outgoing null surface $\lambda=\lambda_{*}$ is employed as a matching surface in our analytic calculations below.

$$
b(v)=\frac{1}{2}\left(\lambda_{\max }(v)+\lambda_{\min }(v)\right) .
$$

Following Ref. [17], we expand the $z$ dependence of all functions in a pseudospectral basis of Chebyshev polynomials. We employ domain decomposition in the $z$ direction with 30 equally spaced domains, each containing eight points.

For the $(\theta, \varphi)$ dependence we employ a basis of scalar, vector and tensor harmonics. These are eigenfunctions of the covariant Laplacian $-\nabla^{2}$ on the unit sphere. The scalar eigenfunctions are just spherical harmonics $y^{\ell m}$. There are two vector harmonics, $\mathcal{V}_{a}^{s \ell m}$ with $s=1,2$, and three symmetric tensor harmonics, $\mathcal{T}_{a b}^{s \ell m}, s=1,2,3$. Explicit representations of these functions are easily found and read [19]

$$
\begin{aligned}
\mathcal{V}_{a}^{1 \ell m} & =\frac{1}{\sqrt{\ell(\ell+1)}} \nabla_{a} y^{\ell m}, \\
\mathcal{V}_{a}^{2 \ell m} & =\frac{1}{\sqrt{\ell(\ell+1)}} \epsilon_{a}{ }^{b} \nabla_{b} y^{\ell m}, \\
\mathcal{T}_{a b}^{1 \ell m} & =\frac{H_{a b}}{\sqrt{2}} y^{\ell m},
\end{aligned}
$$

$$
\begin{aligned}
\mathcal{T}_{a b}^{2 \ell m}= & \frac{1}{\sqrt{\ell(\ell+1)(\ell(\ell+1) / 2-1)}} \epsilon_{(a}{ }^{c} \nabla_{b)} \nabla_{c} y^{\ell m}, \\
\mathcal{T}_{a b}^{3 \ell m}= & \frac{1}{\sqrt{\ell(\ell+1)(\ell(\ell+1) / 2-1)}} \\
& \times\left[\nabla_{a} \nabla_{b}+\frac{\ell(\ell+1)}{2} H_{a b}\right] y^{\ell m},
\end{aligned}
$$

where $\epsilon_{a}{ }^{b}$ has nonzero components $\epsilon_{\theta}{ }^{\varphi}=\csc \theta$ and $\epsilon_{\varphi}{ }^{\theta}=-\sin \theta$, and $H_{a b}=\operatorname{diag}\left(1, \sin ^{2} \theta\right)$ is the metric on the unit sphere. The scalar, vector and tensor harmonics are orthonormal and complete.

We expand the metric and scalar field as follows:

$$
\begin{aligned}
& g_{00}(v, z, \theta, \varphi)=\sum_{\ell m} \alpha^{\ell m}(v, z) y^{\ell m}(\theta, \varphi), \\
& g_{0 a}(v, z, \theta, \varphi)=\sum_{s \ell m} \beta^{s \ell m}(v, z) \mathcal{V}_{a}^{s \ell m}(\theta, \varphi), \\
& g_{a b}(v, z, \theta, \varphi)=\sum_{s \ell m} \gamma^{s \ell m}(v, z) \mathcal{T}_{a b}^{s \ell m}(\theta, \varphi), \\
& \Psi(v, z, \theta, \varphi)=\sum_{\ell m} \chi^{\ell m}(v, z) y^{\ell m}(\theta, \varphi) .
\end{aligned}
$$

Derivatives with respect to $\{\theta, \varphi\}$ can be taken by differentiating the scalar, vector and tensor harmonics.

In order to efficiently transform between real space and mode space, we employ a Gauss-Legendre grid in $\theta$ with $\ell_{\max }+1$ points. Likewise, we employ a Fourier grid in the $\varphi$ direction with $2 \ell_{\max }+1$ points. These choices allow the transformation between mode space and real space to be done with a combination of Gaussian quadrature and fast Fourier transforms.

We truncate the expansions (12) at maximum angular momentum $\ell_{\max }=100$. For axisymmetric simulations we also truncate at azimuthal quantum number $m_{\max }=0$. For nonaxisymmetric simulations we truncate at $m_{\max }=20$.

\section{RESULTS AND DISCUSSION}

We begin by presenting results for axisymmetric simulations. In Fig. 2 we plot the scalar field $\Psi$ as a function of time $v$ and radial coordinate $\lambda$ in the equatorial plane for spin $a=0.9$. The inner and outer boundaries of the shaded region correspond to the curves $\lambda_{\min }(v)$ and $\lambda_{\max }(v)$ and reflect our time-dependent computational domain. As time progresses the scalar wave packet propagates inwards towards $\lambda=1$, becoming increasingly narrower in the process while staying roughly constant in magnitude. As the scalar wave packet approaches $\lambda=1$, the metric at $\lambda>1$ approaches that of Kerr.

The localization of the scalar wave packet to $\lambda=1$ results in large $\lambda$ derivatives of the metric at $\lambda \leq 1$. A useful 


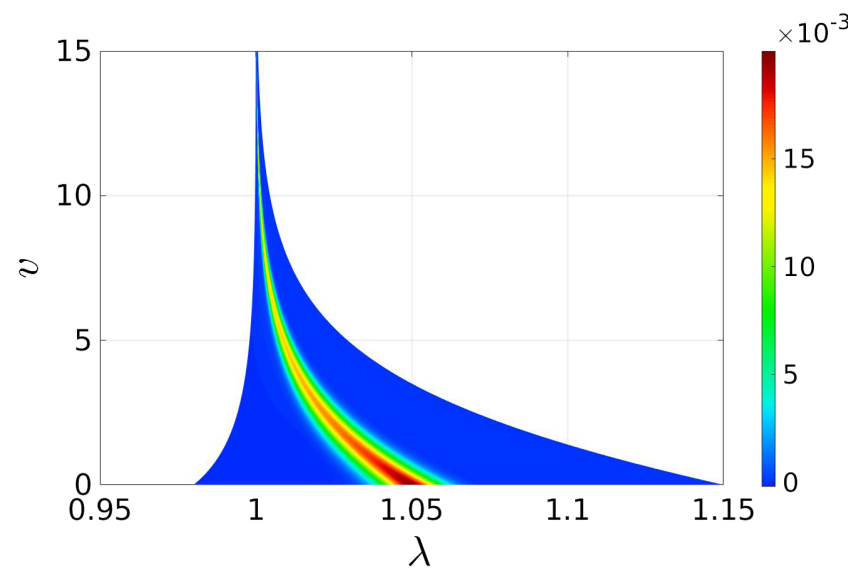

FIG. 2. Evolution of the scalar field $\Psi$ in the equatorial plane for spin $a=0.9$. The inner and outer boundaries of the shaded region represent $\lambda_{\min }(v)$ and $\lambda_{\max }(v)$. As time progresses the scalar field becomes localized at $\lambda=1$.

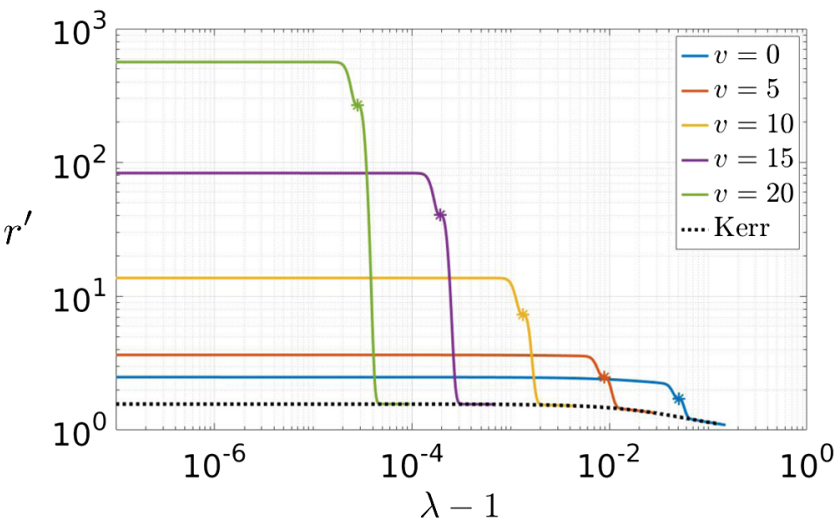

FIG. 3. $r^{\prime}$ in the equatorial plane at several times for $a=0.9$. The *'s denote the locations of the maxima of $\Psi$ at the corresponding times. A shock in $r^{\prime}$ is evident. Outside the shock, $r^{\prime}$ approaches its Kerr value while inside $r^{\prime}$ grows with time.

metric component to study is the areal coordinate $r$, which is related to the volume element via $\sqrt{-g}=r^{2} \sin \theta$. In Fig. 3 we plot $\left.r^{\prime}\right|_{\lambda=1}$ in the equatorial plane at several times, again for spin $a=0.9$. Here and below

$$
{ }^{\prime} \equiv \partial_{\lambda}
$$

The $*$ symbols in the figure denote the maxima of $\Psi$ at the corresponding times. As is evident from the figure, there is a dramatic change in $r^{\prime}$ near the scalar maxima. In other words, there is a shock in $r^{\prime}$. Exterior to the shock $r^{\prime}$ is well approximated by its Kerr value (the dotted curve). The change in $r^{\prime}$ across the shock grows with time.

In Fig. 4 we plot $\left.r^{\prime}\right|_{\lambda=1}$ as a function of $v$ for several values of $\theta$ and for $a=0.9,0.95$ and 0.99 . Also included in each plot is $e^{\kappa v}$ where

$$
\kappa=\frac{1}{2}\left(\frac{1}{M-\sqrt{M^{2}-a^{2}}}-\frac{1}{M}\right)
$$

is the surface gravity of the inner horizon of the corresponding Kerr solution. For $a=0.9,0.95$ and 0.99 we have $\kappa \approx 0.386,0.227$ and 0.0821 , respectively. Our numerics are consistent with the scaling $r^{\prime} \sim e^{\kappa v}$.

We now turn to the curvature. In Fig. 5 we plot the Kretschmann scalar

$$
K \equiv R^{\mu \nu \alpha \beta} R_{\mu \nu \alpha \beta},
$$

as a function of $\lambda$ in the equatorial plane at several times for the same simulations shown in Fig. 2 and 3. The $*$ symbols denote the locations of the maxima of $\Psi$ at the corresponding times. Exterior to the scalar wave packet, $K$ is well approximated by its Kerr value. A prominent feature of Fig. 5 is that $K^{\prime}$ grows dramatically with time just inside the wave packet. In Fig. 6 we plot $\left|K^{\prime}\right|_{\lambda=1}$ as a function of $v$ at several values of $\theta$ for the same simulations shown in Fig. 4. Also included in the plots is $e^{\kappa v}$. Our numerics are consistent with the scaling $\mid K^{\prime} \|_{\lambda=1} \sim e^{\kappa v}$. Evidently, $\lambda=1$ becomes a curvature brick wall at late times, with a shock in $K$ developing there.

The geometry in the vicinity of the shocks can be studied perturbatively [15]. To account for the rapid $\lambda$ dependence, we introduce a bookkeeping parameter $\epsilon \ll 1$ and assume the scalings
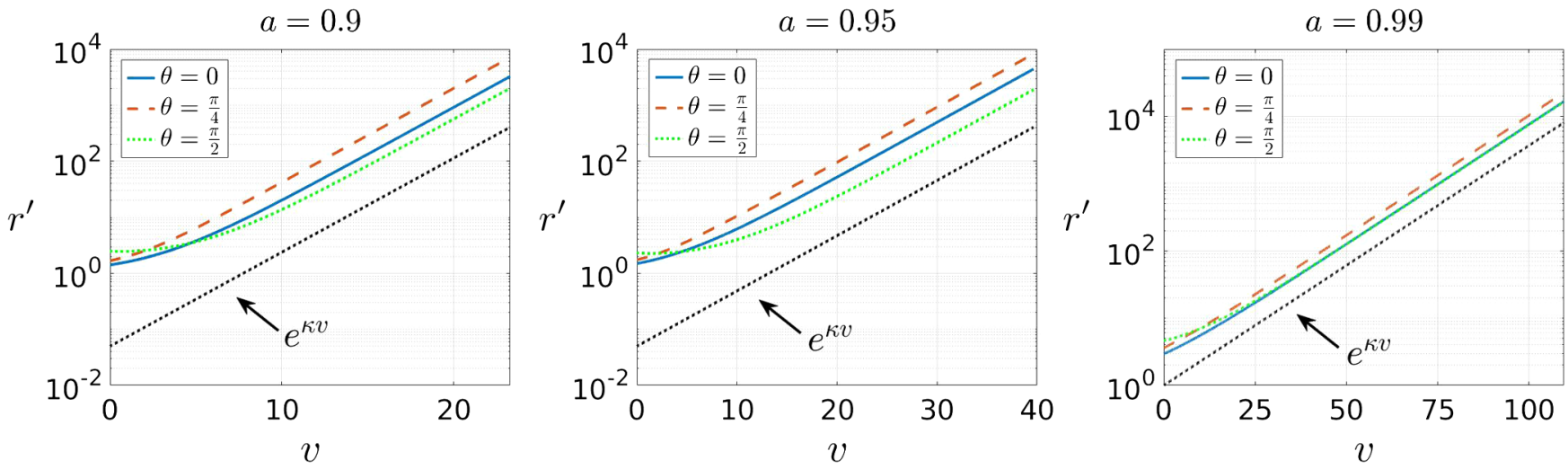

FIG. 4. $\left.\quad r^{\prime}\right|_{\lambda=1}$ for axisymmetric simulations with spin $a=0.9,0.95$ and 0.99 . 


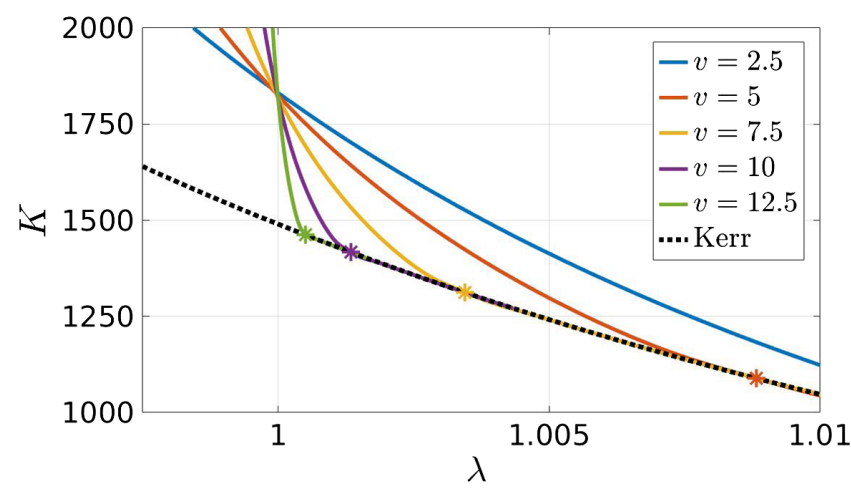

FIG. 5. The Kretschmann scalar $K$ in the equatorial plane at several times for an axisymmetric simulation with $a=0.9$. The *'s denote the locations of the maxima of the scalar wave packet at the corresponding times. Exterior to the wave packet $K$ is well described by its Kerr value. At $\lambda=1 K$ is nearly constant but $K^{\prime}$ grows with time.

$$
\partial_{\lambda} \sim 1 / \epsilon, \quad A \sim \epsilon .
$$

The scaling $A \sim \epsilon$ is necessary to have $A^{\prime}=O(1)$, which itself is necessary to have a finite surface gravity at $\lambda=1$ as $v \rightarrow \infty$. We then solve the Einstein-scalar system in the region $\lambda<\lambda_{*}$ with $\lambda_{*}=1+O(\epsilon)$. A convenient choice of matching surface $\lambda=\lambda_{*}$ is an outgoing null sheet exterior to the scalar wave packet, as shown in Fig. 1.

On the surface $\lambda=\lambda_{*}$ we impose the boundary condition that the geometry is that of Kerr and that the scalar field vanishes. At leading order in $\epsilon$ we therefore need the inner horizon values of the Kerr metric. In our coordinate system, at the inner horizon of Kerr we have

$\left.A\right|_{\lambda=1}=0,\left.\quad A^{\prime}\right|_{\lambda=1}=-\kappa,\left.\quad F^{a}\right|_{\lambda=1}=\Omega \delta^{a \phi}$,

where

$$
\Omega=\frac{\sqrt{M^{2}-a^{2}}+M}{2 a M},
$$
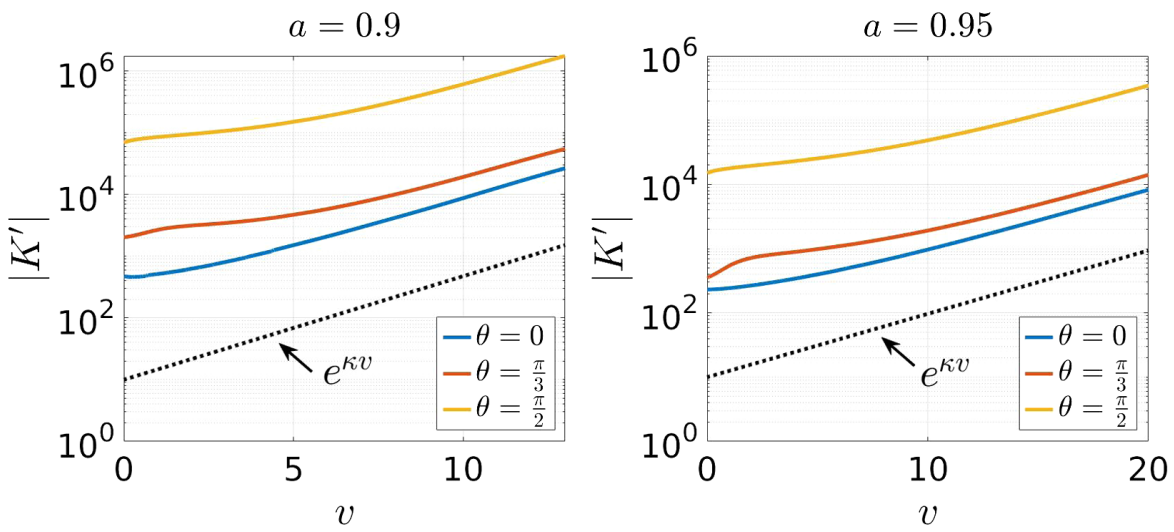

is the angular velocity of the inner horizon. It follows that for the Kerr solution

$$
\left.D_{+} g_{\mu \nu}\right|_{\lambda=1}=0
$$

where

$$
D_{+} \equiv \partial_{v}+A \partial_{\lambda}+F^{a} \partial_{a}
$$

is the directional derivative along outgoing null geodesics.

At leading order in $\epsilon$ the $(\mu, \nu)=(v, a)$ component of the Einstein equations (1) reduces to

$$
F^{\prime a}=0 .
$$

The boundary conditions (17) therefore imply

$$
F^{a}=\Omega \delta^{a \phi} .
$$

With the solution (22), at leading order in $\epsilon$ the $(\mu, \nu)=$ $(v, \lambda)$ and $(a, b)$ components of Einstein's equations reduce to $[20]$

$0=\left(r D_{+} r\right)^{\prime}$,

$0=\left[\delta_{a}^{c} \delta_{b}^{d}-\frac{1}{2} h^{c d} h_{a b}\right]\left(r D_{+} h_{c d}\right)^{\prime}+D_{+} r h_{a b}^{\prime}$,

$0=A^{\prime \prime}+\frac{1}{4} h^{a b} h_{b c}^{\prime} h^{c d} D_{+} h_{d a}-\frac{2 r^{\prime} D_{+} r}{r}+8 \pi \Psi^{\prime} D_{+} \Psi$,

where $h^{a b}$ is the inverse of $h_{a b}$. Likewise, at leading order in $\epsilon$ the scalar equation of motion (2) reduces to

$$
\left(r D_{+} \Psi\right)=-\Psi^{\prime} D_{+} r .
$$

Equations (23a), (23b) and (24) are just radial wave equations for $r, h_{a b}$ and $\Psi$. Imposing the boundary condition that the geometry exterior to the shell is that of Kerr is tantamount to imposing the boundary condition

FIG. 6. $\left|K^{\prime}\right|_{\lambda=1}$ evaluated at several polar angles for axisymmetric simulations with spin $a=0.9,0.95$ and 0.99 . 
that there is no infalling radiation through the shell, which is what Eq. (19) states. With the boundary condition (19), Eqs. (23a), (23b) and (24) have the solutions

$$
D_{+} r=0, \quad D_{+} \Psi=0, \quad D_{+} h_{a b}=0 .
$$

These first-order wave equations state that excitations in $r$, $\Psi$ and $h_{a b}$ are transported along outgoing null geodesics tangent to $D_{+}$. Substituting Eq. (25) into Eq. (23c) and employing the boundary conditions (17), we secure

$$
A=-\kappa \lambda
$$

With the solutions (26) and (22) and the definition of $D_{+}$ in Eq. (20), the first-order system (25) is solved by

$$
\begin{gathered}
r(v, \lambda, \theta, \varphi)=\rho\left(e^{\kappa v}(\lambda-1), \theta, \varphi-\Omega v\right), \\
\Psi(v, \lambda, \theta, \varphi)=\psi\left(e^{\kappa v}(\lambda-1), \theta, \varphi-\Omega v\right), \\
h_{a b}(v, \lambda, \theta, \varphi)=\mathcal{H}_{a b}\left(e^{\kappa v}(\lambda-1), \theta, \varphi-\Omega v\right),
\end{gathered}
$$

where $\rho, \psi$ and $\mathcal{H}_{a b}$ are arbitrary functions. Note that curves with $e^{\kappa v}(\lambda-1), \theta$, and $\varphi-\Omega v$ all constant are simply outgoing null geodesics near $\lambda=1$. These geodesics spiral in towards $\lambda=1$ at angular frequency $\Omega$, which is due to frame dragging, and eventually terminate at $\lambda=1$ as $v \rightarrow \infty$. The value of the fields on these geodesics is constant. Since the $\lambda$ dependence comes in the combination $e^{\kappa v}(\lambda-1)$, it follows that $e^{-\kappa v}$ plays the role of our bookkeeping parameter $\epsilon$.

The above analysis implies that as $v \rightarrow \infty$, the scalar wave packet must approach $\lambda=1$, as seen in Fig. 2 . Moreover, it immediately follows from Eq. (27) that the individual components of the Riemann tensor scale like

$$
R_{\mu \nu \alpha \beta} \sim e^{2 \kappa v} .
$$

Indeed, in Fig. 7 we plot the component $\left.R_{v \lambda v \lambda}\right|_{\lambda=1}$ in the equatorial plane for an axisymmetric simulation with spin $a=0.9$ and verify this scaling. The exponential growth in Eq. (28) reflects the fact that outgoing radiation is blueshifted by a factor of $e^{\kappa v}$, becoming exponentially localized in $\lambda$ in the process. However, owing to the fact that all excitations in Eq. (27) are purely outgoing, the curvature scalar $K$ cannot blow up exponentially, meaning all exponential factors in Eq. (15) cancel. Why must this happen? Since by construction there is no infalling radiation present, one can simply boost to the frame where the outgoing radiation is not blueshifted and the components $R_{\mu \nu \alpha \beta}$ and Kretschmann scalar are finite as $v \rightarrow \infty$. Simply put, with only outgoing radiation present, the Kretschmann scalar - and in fact all other scalars - can only depend on $\{v, \lambda, \varphi\}$ through the combinations $e^{\kappa v}(\lambda-1)$ and $\varphi-\Omega v$. It therefore follows that $K$ is finite on the shocks and that

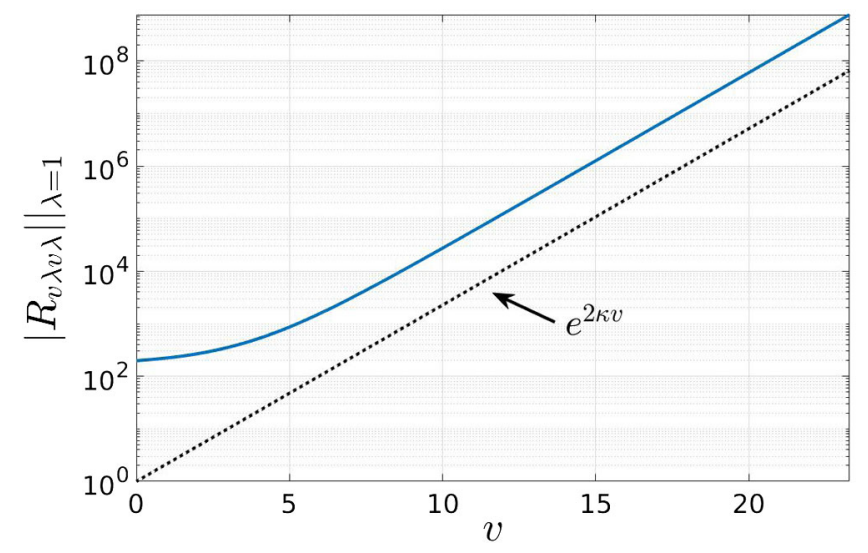

FIG. 7. The Riemann tensor component $\left.R_{v \lambda v \lambda}\right|_{\lambda=1}$ in the equatorial plane for an axisymmetric simulation with spin $a=0.9$. The Riemann tensor diverges like $e^{2 \kappa v}$.

$\left.r^{\prime}\right|_{\lambda=1}=e^{\kappa v} H(\theta, \varphi-\Omega v),\left.\quad K^{\prime}\right|_{\lambda=1}=e^{\kappa v} Q(\theta, \varphi-\Omega v)$,

for some functions $H$ and $Q$. The scaling relations (29) match those shown in Figs. 4 and 5 for our axisymmetric simulations.

The scaling relations (29) also demonstrate that rotation invariance in $\varphi$ can be broken: a small nonaxisymmetric perturbation in initial data results in violations of axisymmetry in $r^{\prime}$ and $K^{\prime}$ which are exponentially amplified. To demonstrate this, in Fig. 8 we plot $\left.K^{\prime}\right|_{\lambda=1}$ at $\theta=\pi / 2$ as a function of time for a nonaxisymmetric simulation with $a=0.95$. The left figure is evaluated at $\varphi=0$ while the right figure is evaluated at $\varphi=\Omega v$. At $\varphi=0$ we see that $K^{\prime}$ grows exponentially with sinusoidal oscillations superimposed. In the rotating frame, where $\varphi=\Omega v$, the sinusoidal oscillations are not present, just as Eq. (29) requires. Evidently, the curvature brick wall at $\lambda=1$ retains the angular structure contained in the initial data. Oscillating features of the curvature were also reported in Ref. [21].

Let us now turn to analyzing the effect of the shocks on infalling geodesics. Consider first radial infalling null
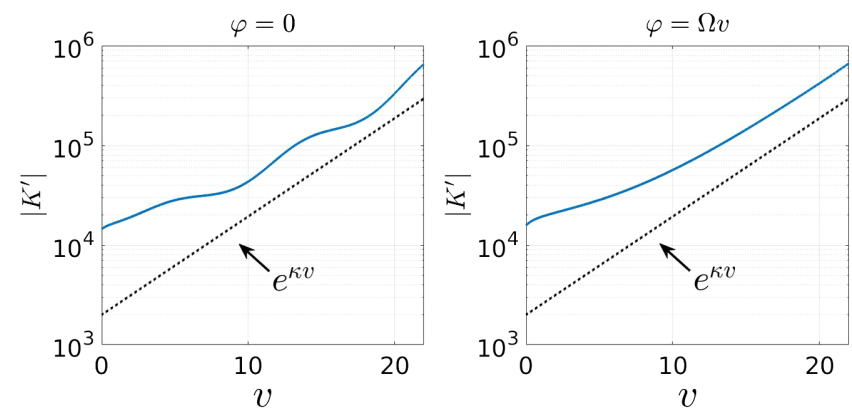

FIG. 8. $\left|K^{\prime}\right|_{\lambda=1}$ in the equatorial plane for a nonaxisymmetric simulation with spin $a=0.95$. The left plot is evaluated at $\varphi=0$ whereas the right plot is evaluated at $\varphi=\Omega v$. 
geodesics with $v=$ const. The null energy condition implies that $r^{\prime \prime} / r<0$. This means that $r^{\prime}$ can only increase as $\lambda$ decreases. Since just inside the shock $r^{\prime} \sim e^{\kappa v}$, it follows that the affine distance $\Delta \lambda$ from the shock to the point $r=0$ is

$$
\Delta \lambda \sim e^{-\kappa v}
$$

This reflects the fact that the shock focuses infalling light rays to $r=0$, which can also be seen from Raychaudhuri's equation. Turning now to infalling timelike geodesics, the scaling (28) means that infalling timelike observers crossing the shocks will experience tidal forces of order $e^{2 \kappa v}$. In particular, upon crossing the shocks the areal velocity will be

$$
\frac{d r}{d \tau} \sim-e^{\kappa v} .
$$

What then is the fate of an observer who jumps into the black hole at late times? For large enough black holes, observers need not experience any ill effects until they pass through the shocks. They will measure the local geometry to be that of Kerr, with arbitrarily small tidal forces. Upon encountering the shocks though, they will be torn apart by tidal forces and their subsequent debris will be accelerated nearly to the speed of light towards the black hole interior.
In the present paper we only considered perturbations in the interior of black holes and did not allow infalling radiation. Exterior perturbations of black holes in asymptotically flat spacetime result in infalling radiation which decays with a power law in $v$ in accord with Price's law [22]. For Reissner-Nordström black holes, infalling radiation results in a weak null curvature singularity developing on the ingoing leg of the inner horizon ( $v=\infty$ in Fig. 1) [5,6]. A similar effect should happen for Kerr black holes. With infalling radiation, the exponential factors in Eq. (28) cannot be ameliorated via a boost, for a boost which compensates the $e^{\kappa v}$ blueshift of outgoing radiation will inevitably result in the infalling radiation being blueshifted by a factor of $e^{\kappa v}$. With infalling radiation present, it is therefore reasonable to expect that $K$ will blow up like $e^{2 \kappa v}$. We leave the inclusion of infalling radiation for future studies.

\section{ACKNOWLEDGMENTS}

This work was supported by the Black Hole Initiative at Harvard University, which is funded by a grant from the John Templeton Foundation. E. C. is also supported by Grant No. 312032894 from the Deutsche Forschungsgemeinschaft. We thank Amos Ori and Peter Galison for useful comments and discussions.
[1] R. Penrose, Structure of space-time, Published in Battelle Rencontres, edited by C. M. DeWitt and J. A. Wheeler, Lectures in Mathematics and Physics (Benjamin, New York, 1968).

[2] M. Simpson and R. Penrose, Internal instability in a Reissner-Nordstrom black hole, Int. J. Theor. Phys. 7, 183 (1973).

[3] W. A. Hiscock, Evolution of the interior of a charged black hole, Phys. Lett. 83A, 110 (1981).

[4] Y. Gürsel, I. D. Novikov, V. D. Sandberg, and A. A. Starobinsky, Final state of the evolution of the interior of a charged black hole, Phys. Rev. D 20, 1260 (1979).

[5] E. Poisson and W. Israel, Inner-Horizon Instability and Mass Inflation in Black Holes, Phys. Rev. Lett. 63, 1663 (1989).

[6] E. Poisson and W. Israel, Internal structure of black holes, Phys. Rev. D 41, 1796 (1990).

[7] A. Ori, Inner Structure of a Charged Black Hole: An Exact Mass-Inflation Solution, Phys. Rev. Lett. 67, 789 (1991).

[8] M. L. Gnedin and N. Y. Gnedin, Destruction of the cauchy horizon in the reissner-nordstrom black hole, Classical Quantum Gravity 10, 1083 (1993).

[9] P. R. Brady and J.D. Smith, Black Hole Singularities: A Numerical Approach, Phys. Rev. Lett. 75, 1256 (1995).
[10] L. M. Burko, Structure of the Black Hole's Cauchy Horizon Singularity, Phys. Rev. Lett. 79, 4958 (1997).

[11] S. Hod and T. Piran, Mass Inflation in Dynamical Gravitational Collapse of a Charged Scalar Field, Phys. Rev. Lett. 81, 1554 (1998).

[12] L. M. Burko and A. Ori, Analytic study of the null singularity inside spherical charged black holes, Phys. Rev. D 57, R7084 (1998).

[13] E. Eilon and A. Ori, Numerical study of the gravitational shock wave inside a spherical charged black hole, Phys. Rev. D 94, 104060 (2016).

[14] M. Dafermos, Stability and instability of the cauchy horizon for the spherically symmetric einstein-maxwell-scalar field equations, Ann. Math. 158, 875 (2003).

[15] D. Marolf and A. Ori, Outgoing gravitational shock-wave at the inner horizon: The late-time limit of black hole interiors, Phys. Rev. D 86, 124026 (2012).

[16] More precisely, the shocks form just outside where the outgoing branch of the inner horizon would be in the Reissner-Nordström geometry.

[17] P. M. Chesler and L. G. Yaffe, Numerical solution of gravitational dynamics in asymptotically anti-de Sitter spacetimes, J. High Energy Phys. 07 (2014) 086. 
[18] P. M. Chesler and D. A. Lowe, Nonlinear evolution of the $\mathrm{AdS}_{4}$ black hole bomb, arXiv:1801.09711.

[19] V. D. Sandberg, Tensor spherical harmonics on $s^{2}$ and $s^{3}$ as eigenvalue problems, J. Math. Phys. (N.Y.) 19, 2441 (1978).

[20] The remaining components of Einstein's equations, the $(\mu, \nu)=(v, v),(\lambda, \lambda)$ and $(\lambda, a)$ components, are initial value and radial constraint equations, respectively, and will not be necessary for our analysis here.

[21] A. Ori, Oscillatory Null Singularity inside Realistic Spinning Black Holes, Phys. Rev. Lett. 83, 5423 (1999).

[22] R. H. Price, Nonspherical perturbations of relativistic gravitational collapse. II. Integer-Spin, zero-rest-mass fields, Phys. Rev. D 5, 2439 (1972). 